\title{
A New Method for Promoting Biologic Synthesis and Reducing the Size of Titanium Dioxide Nanoparticles (Tio2 NPS) Synthesized by Origanum Vulgare
}

\section{Mahmoud Bahmani ${ }^{1 *}$}

${ }^{1}$ Biotechnology and Medicinal Plants Research Center, Ilam University of Medical Sciences, Ilam, Iran

Article Info

*Correspondence to: Mahmoud Bahmani

mahmood.bahmani@gmail.com

Article History:

Received: 12 August 2019

Accepted: 15 Oct 2019

ePublished: : 20 Nov 2019

Keywords: Titanium dioxide, Nanoparticles (Tio2 NPs), Origanum vulgare

\section{Abstract}

As it is known the particle size is the most important issue in the preparation of nanoparticles. Nowadays, conventional drug therapy is characterized by limited effectiveness and undesirable side effects which are due to the lack of selectivity and poor distribution. Hence, reduction of particle size is considered as an approach to reduce toxicity, increase release and bioavailability, improve solubility, and produce a better formulation for a higher quality. Therefore, reduction of the particle size of nanoparticles is important. We would like to present a new method we used to reduce the particle average size to $64 \mathrm{~nm}$. In the study of Sankar et al. an aqueous extract was used to prepare the nanoparticle and the size of the titanium dioxide nanoparticle synthesized with aqueous Origanum vulgare extract in a Dynamic Light Scattering Analysis was $341 \mathrm{~nm}$. We used hydroalcoholic extract and prepared titanium dioxide nanoparticles by using O. vulgare hydroalcoholic extract. The size of prepared nanoparticles was $64 \mathrm{~nm}$ which is about one-fifth of the nanoparticle sizes produced in Renu Sankar et al. study.

\section{How to cite this paper}

Bahmani M. A new method for promoting biologic synthesis and reducing the size of titanium dioxide nanoparticles (Tio2 NPs) synthesized by Origanum vulgare. Plant Biotechnology Persa 2019; 1(1): 10-12.

\section{Dear editor;}

As you know the particle size is the most important issue in preparation of nanoparticles. In fact nanoparticles are usually characterized and evaluated by three factors including nanoparticle size distribution and morphology, particle size being the most important factor. The size of nanoparticle has substantial effects on drug release and drug delivery. Smaller sizes of nanoparticles which enhance the surface areas, significantly increase drug release. Furthermore, it has been suggested that degradation of polymers might be affected by the size of nanoparticle and there is a compromise between small particle size in nanoparticles and maximum stability [1,2].Nowadays, conventional drug therapy is characterized by limited 
effectiveness and undesirable side effects which are due to lack of selectivity and poor distribution. Hence, delivering the therapeutic materials to a desirable site is one of the most important issues in management of diseases. Enhancing the selective drug delivery undoubtedly reduces these limitations through delivering drug to the action site and enhancing drug concentration in a target tissue. This also reduces the side effects of drug by reducing the required 43 drug doses. Such type of therapy is required when there is a discrepancy between the dose of a drug and its therapeutic results or toxic effects. Hence, reduction of particle size is considered as an approach to reduce toxicity, increase release and bioavailability, improve solubility and produce a better formulation for a higher quality. Therefore, reduction of the particle size of nanoparticles is important. In a manuscript entitled "Wound healing activity of Origanum vulgare engineered titanium dioxide nanoparticles in Wistar albino rats" by Sankar et al. published in 2014 [3], the aqueous extract was used to prepare the nanoparticle and the size of the titanium dioxide nanoparticle synthesized with aqueous marjoram extract in a Dynamic Light Scattering Analysis was $341 \mathrm{~nm}$. We would like to present a new method we used to reduce the particle average size to 64 $\mathrm{nm}$. In the study of Sankar et al. an aqueous extract was used to prepare the nanoparticle and the size of the titanium dioxide nanoparticle synthesized with aqueous marjoram extract in a Dynamic Light Scattering Analysis was $341 \mathrm{~nm}$. We used hydroalcoholic extract and prepared titanium dioxide nanoparticles by using marjoram hydroalcoholic extract. The size of nanoparticles we prepared were much smaller average size $(64 \mathrm{~nm})$, which are about one-fifth of the nanoparticle sizes produced in Renu Sankar et al. study. We believe the main reason for this is related to ethanol, because alcoholic solvents are more capable of extracting all the substances compared to aqueous solvent (Fig. 1).
Figure 1. Scaning electron microscope (SEM) of titanium dioxide nanoparticles synthesized with Origanum vulgare hydroalcoholic extract

The method we used for preparation of hydroalcoholic extract was as follow: $200 \mathrm{~g}$ of powder of each plant was mixed with ethanol (Nasr, Iran) and distilled water (70:30). After $4 \mathrm{~h}$ shaking, the herbal solution was left in the laboratory for $24 \mathrm{~h}$. Then, the mixture was passed through a filter paper, and the solvent was isolated from the extract using a rotary evaporator (IKA ${ }^{\star}$ RV10) under vacuum condition at $70^{\circ} \mathrm{C}$ and $150 \mathrm{rpm}$. The concentrated extract of the plant was poured into the plate and dried. For green synthesis of titanium dioxide (Tio2) nanoparticles (NPs), $1000 \mathrm{mg}$ of hydroalcoholic $\mathrm{H}$. perforatum, O. vulgare extracts and $30 \mathrm{mg}$ of carvacrol or hypericin were added to $90 \mathrm{~mL}$ of titanium isopropoxide (with $5 \mathrm{mM}$ ) in an Erlenmeyer flask with shaker. Each 30 min of shaking the solution, it was increased to $5 \mathrm{~min}$ at $50^{\circ} \mathrm{C}$ to synthesize the materials and compounds. Afte $5 \mathrm{~h}$ of continuous stirring, the solution obtained in $50 \mathrm{~mL}$ falcons was centrifuged at $12000 \mathrm{rpm}$ at $4^{\circ} \mathrm{C}$ for $15 \mathrm{~min}$. This was continued until the supernatant became transparent and the synthesized materials completely deposited. After drying the centrifuged solution, the obtained compound was used for the laboratory tests.

\section{Authors' contribution}

All authors contributed equally to the manuscript.

\section{Conflicts of interest}

The authors declared no competing interests.

\section{Ethical considerations}

Ethical issues (including plagiarism, data fabrication, double publication and etc.) have been completely observed by author.

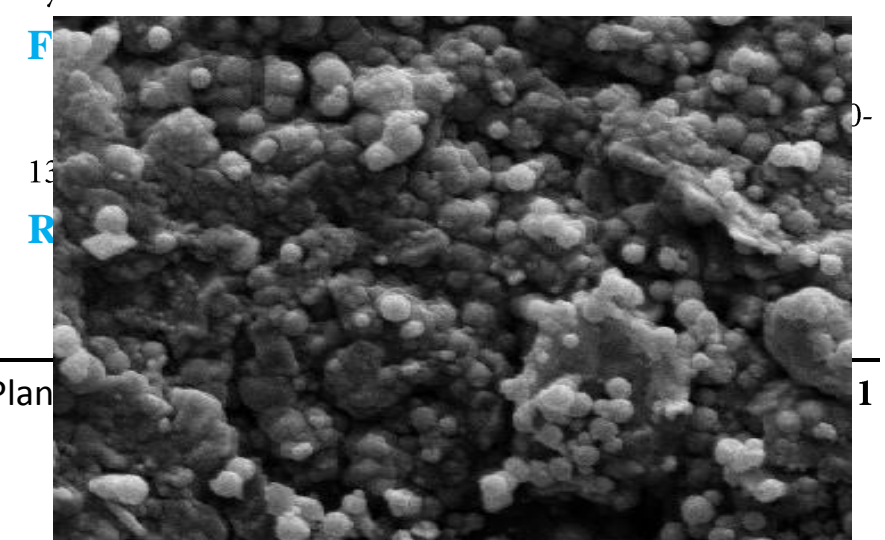


1. [1] Redhead HM, Davis SS, Illum LJ. Control. Release. 2001; 70: 353-8.

2. [2] Betancor L, Luckarift HR. Trends Biotechnol. 2008; 26: 566.

3. [3] Sankar R, Dhivya R, Shivashangari KS, Ravikumar V. Wound healing activity of Origanum vulgare engineered titanium dioxide nanoparticles in Wistar Albino rats. J Mater Sci Mater Med. 2014; 25(7):17018 